\title{
Modelação longitudinal dos níveis de coordenação motora de crianças dos seis aos 10 anos de idade da Região Autônoma dos Açores, Portugal
}

CDD. 20.ed. 152.385

\author{
Renata Karine Batista Coelho de DEUS* \\ Alcibíades BUSTAMANTE**** \\ Vitor Pires LOPES ${ }^{* * *}$ \\ André Teixeira e SEABRA* \\ Rui Manuel Garganta da SILVA* \\ José António Ribeiro MAIA*
}

*Faculdade de Desporto, Universidade do Porto - Portugal. ${ }^{* *}$ Universidade $\mathrm{Na}$ cional de Educación "Enrique Guzmán y Valle" - La Cantuta Lima - Peru.

***Departamento de Ciências do Desporto, Instituto Politécnico de Bragança - Portugal.

\section{Resumo}

Este estudo é percorrido por três principais objetivos: 1) caracterizar o desenvolvimento modal e as diferenças inter-individuais da coordenação motora; 2) verificar a existência de um efeito associado ao gênero; 3) testar a relevância de preditores do desenvolvimento da Coordenação motora (CoM) tais como o IMC e os níveis de Atividade Física. A amostra é constituída de 285 crianças (143 meninos e 142 meninas). A coordenação motora foi determinada a partir da bateria de testes KTK (Equilíbrio em deslocamento para trás, Saltos laterais, Saltos monopedais e Transposição lateral). 0 questionário de Godin e ShepHARD (1985) foi utilizado para avaliar a Atividade Física. As estatísticas descritivas básicas foram calculadas no SPSS 15. A mudança intra-individual e as diferenças inter-sujeitos foram efetuadas no programa estatístico HLM, versão 6. É visivel um incremento dos valores médios, em ambos os sexos, para as todas as provas do KTK, bem como do IMC e o dos niveis de Atividade Física. Os melhores resultados foram observados nas crianças que estavam no $1^{\circ}$ quartil do IMC e no $3^{\circ}$ da Atividade Física. Os resultados reafirmam a necessidade de uma estrutura didático-metodologica das aulas de Educação Física para que as crianças alcancem níveis adequados de coordenação para sua idade.

UnIteRmos: Coordenação motora; Crianças; Estudo longitudinal; Modelação hierárquica.

\section{Introdução}

Uma parte substancial da pesquisa desenvolvimentista da coordenação motora $(\mathrm{CoM})$ situada no contexto escolar com crianças do $1^{\circ}$ Ciclo do Ensino Básico (CEB) tem-se preocupado, sobretudo, em descrever as diferenças de médias entre sexos ao longo da idade, bem como as respectivas interações a partir de delineamentos de natureza transversal (LOPES, 1997). A leitura exclusiva das médias, não obstante centrar-se na análise da variância (ANOVA), é algo limitada. Este tipo de abordagem não permite a construção consistente de um discurso mais elaborado sobre aspectos da verdadeira mudança dos níveis da CoM, uma vez que somente delineamentos de natureza longitudinal podem responder às diferentes facetas associadas à mudança que ocorre no tempo. Daqui a necessidade de construir modelos que descrevam a complexidade de diferentes aspectos da mudança do desempenho da CoM em função do tempo para além da sua leitura modal. A mudança pode expressar-se por trajetórias distintas refletindo uma forte variação inter-individual no comportamento intra-individual. É também de esperar que haja incrementos na CoM ao longo da idade que não sejam da mesma magnitude em meninos e meninas, tão pouco em momentos adjacentes no tempo.

A informação longitudinal centrada no desenvolvimento da CoM de crianças e jovens não é tão extensa quanto se desejaria. Inexplicavelmente, pelo menos 
do ponto de vista pedagógico, os dados de pesquisas longitudinais puras com mais de dois pontos no tempo são relativamente "antigos", isto é, têm cerca de 30 anos e devem-se ao trabalho de WilLimcziK (1980). Este autor realizou um estudo longitudinal com 399 crianças e constatou um crescimento linear na prestação dos testes da bateria do KTK (Körperkoordinationstest für Kinder) entre os 6,7 e os 10,7 anos de idade. Os resultados sugerem que muito provavelmente as aulas de Educação Física (EF) contribuíram para que tal acontecesse, confirmando, uma vez mais, a sua relevância educativa no desenvolvimento da capacidade de coordenação corporal. GRAF, KoCH, Kretschmann-Kandel, Falkowski, Christ, Coburger, Lehmacher, BJarnason-Wehrens, Platen, Tokarski, Predel e Dordel (2004), num estudo de caso-controle, como parte de um programa de intervenção nas escolas primárias alemãs, combinou aspectos da educação para a saúde com a atividade física (AtFís), analisando a influência do índice de massa corporal (IMC) na CoM que foi avaliada pela bateria KTK. Os valores na prova de saltos laterais (SL) foram significativamente mais elevados em crianças com o IMC normal em relação às que apresentavam sobrepeso e obesidade. Posteriormente, os mesmos autores (Graf, Koch, FalKowski, Jouck, Christ, Stauenmaier, BJarnason-Wehrens, Tokarski, Dordel \& Predel, 2005) numa outra investigação verificaram que o quociente motor $(\mathrm{QM})$, de crianças com sobrepeso e obesidade, apresentava valores inferiores aos de crianças normo-poderais e de baixo peso para a sua idade. LOPES (1997) analisou a mudança ocorrida ao longo do ano letivo na CoM de crianças em idade escolar sujeitas a diferentes programas e a diferentes frequências semanais de aulas de EF ao longo de um ano escolar. Constatou mudanças significativas díspares nos níveis de expressão da capacidade de CoM quando sujeitas a programas distintos de aulas de EF, isto é, as aulas tiveram efeitos positivos no desenvolvimento da CoM.

Tal como referimos anteriormente, para além do estudo de WiLLIMCZIK (1980) não conseguimos

\section{Metodologia}

\section{Amostra}

A amostra constituída por 285 crianças (142 meninas e 143 meninos) é um sub-conjunto amostral de um vasto estudo auxológico e epidemiológico identificar qualquer outra pesquisa de natureza longitudinal pura centrada em crianças do $1^{\circ} \mathrm{Ciclo}$ do Ensino Básico (CEB). Esta falta de informação acerca do desenvolvimento coordenativo de crianças dos seis aos 10 anos é algo incompreensível, originando preocupações acerca do significado e do valor de opções didático-metodológicas de planos de aulas ou orientações dos programas nacionais de Educação Física para o $1^{\circ}$ ciclo (LOPES, MaIA, Silva, Seabra \& Moraes, 2003). Esta ausência reclama um olhar cuidadoso e atento para se entender aspectos essenciais do desenvolvimento da CoM de crianças do $1^{\circ} \mathrm{CEB}$. Uma forma indireta de mapear aspectos da mudança que ocorre na CoM radica no uso de baterias de testes de fácil aplicação. A bateria de testes KTK tem sido utilizada com frequência em países lusófonos (ANDRADE, 1996; GOMES, 1996; LOPES, 1997; LOPES \& MAIA, 1997; LOPES et al., 2003; MоTA, 1991; SiLVA, 1989), apresentando informação de utilidade aos professores de Educação Física no $1^{\circ} \mathrm{CEB}$, apesar de não estar ainda contida nos programas oficiais da disciplina de Educação Físico-Motora do $1^{\circ}$ CEB. Estudar o comportamento modal e diferenças inter-individuais a que se associam preditores da mudança são problemas não sanados. Decorre daqui o desafio do presente trabalho ao utilizar recursos de análise da Modelação Hierárquica (sobre esta metodologia, e em língua portuguesa, ver Maia, Lopes, Silva, Seabra, FerreiRA \& CARDOSO, 2003; em língua inglesa consultar Goldstein, 1987, 1995).

O estudo que passaremos a descrever pretende mostrar aspectos da "história natural" do desenvolvimento da CoM de crianças do $1^{\circ} \mathrm{CEB}$ Português a partir de um delineamento longitudinal puro. Daqui que os seus propósitos sejam os seguintes: 1) caracterizar o desenvolvimento modal e as diferenças inter-individuais da CoM; 2) verificar se existe um efeito associado ao gênero, favorecendo os meninos; 3) testar a relevância de preditores do desenvolvimento da CoM que também se alteram no tempo - o IMC e os níveis de AtFís. do desenvolvimento motor realizado na Região Autônoma dos Açores (RAA) que foi desenhado, essencialmente, para avaliar aspectos somato-motores de crianças e jovens no intervalo etário dos seis aos 19 anos com base num delineamento longitudinal-misto 
(Maia \& Lopes, 2003). A amostra está repartida por quatro ilhas (Faial, Pico, São Miguel e Terceira), sendo constituída por quatro coortes. Para o presente estudo foram consideradas apenas as crianças da $1^{\text {a }}$ coorte acompanhadas dos seis aos 10 anos de idade. É importante salientar que não foi possível obter informação completa de todas as crianças (circunstância corrente em estudos com esta dimensão). De 285 crianças avaliadas, o número de casos omissos variou entre 10\% e 18\% (teste SM e questionário do nível de AtFís respectivamente). Nenhum destes casos omissos tem um padrão específico que condicione as estimativas dos parâmetros dos modelos a serem testados. É importante referir que no domínio da Modelação Hierárquica a informação omissa não representa um problema de análise, uma vez que esta metodologia estatística otimiza toda a informação disponível.

\section{Avaliação da coordenação motora}

A avaliação da CoM foi realizada através da bateria de teste KTK (Körperkoordinationstest für Kinder) que foi desenvolvida em 1974 pelos pesquisadores alemães KiPHARD e SCHILLING (1974) considerando uma abordagem simultaneamente clínica e pedagógica. $\mathrm{O}$ objetivo principal da bateria era, e ainda continua a ser, o de identificar crianças com alguma insuficiência no desenvolvimento coordenativo a partir da classificação do valor do quociente motor (QM). Esta bateria é constituída por quatro testes: 1) Equilíbrio em deslocamento para trás (EDT);2) saltos laterais (SL); 3) saltos monopedais (SM); e 4) transposição lateral (TL). Neste estudo, os resultados das quatro provas serão analisados individualmente por forma de obter uma visão mais detalhada do desempenho coordenativo. Em nenhum momento deste texto será utilizado o valor do QM por questôes que se prendem com a insuficiência de conhecimento da sua validade transcultural. Uma descrição detalhada dos testes da bateria KTK pode ser encontrada em MAIA e LoPES (2003).

\section{Medidas antropométricas}

A altura foi medida com um estadiômetro entre o vértex e o plano de referência do solo. $\mathrm{O}$ resultado foi anotado em centímetros $(\mathrm{cm})$. As crianças foram pesadas com a menor quantidade de roupa possível (camiseta e "short") em uma balança com registos de 100 gramas. $\mathrm{O}$ resultado foi anotado em $\mathrm{kg}$ com aproximaçāo ao hectograma. O IMC foi calculado de acordo com a fórmula bem conhecida, $\mathrm{IMC}=$ peso $(\mathrm{kg}) / \mathrm{altura}^{2}(\mathrm{~m})$.

\section{Avaliação da atividade física}

O questionário de Godin e Shephard (1985), desenvolvido no Canadá, foi utilizado para avaliar a AtFís semanal. O questionário pretende identificar a AtFís realizada na ocupação dos tempos livres durante sete dias. Os sujeitos registram o número de vezes, por semana, em que despendem mais de 15 minutos em atividades que são classificadas como leves (3 METs), moderadas (5 METs) ou extenuantes (9 METs). O "score" final obtém-se pela multiplicação da frequência em cada categoria pelo valor de MET respectivo e pela soma posterior dos produtos obtidos.

\section{Procedimentos estatísticos}

A análise da mudança intra-individual e das diferenças inter-sujeitos foi efetuada com o programa estatístico HLM, versão 6 (RAUdenduUsh, BRYK, Cheong \& Congdon, 2004), que permite testar, sequencialmente, diferentes modelos de complexidade crescente que melhor representam o comportamento dos dados longitudinais (para referências em língua portuguesa ver Maia, Garganta, Seabra, Lopes, Vinagre, Freitas, Prista \& Meira, 2005; Maia, Sousa, Fermino, Seabra, Silva, Silva, Bustamante, Lopes, Prista \& Freitas, 2007; Maia et al., 2003). Nesta metodologia analítica, o primeiro nível é composto pelos registros repetidos no tempo nos quatro testes de CoM, bem como pelos preditores cujos valores também mudam no tempo - IMC e AtFís; no segundo nível, i.e., o dos sujeitos, foi considerado somente o gênero como covariável que pode induzir a presença de trajetórias modais distintas. A avaliação sequencial dos modelos, hierarquicamente contidos e de complexidade crescente (por adição de parâmetros), foi efetuada a partir da estatística Deviance e da sua redução (cujo valor se espera significativo, $\mathrm{p}<0,05$ ).

A estruturação sequencial dos modelos para interpretar a variância e covariância existente nos dois planos da hierarquia informacional foi a seguinte:

1) Para determinar a quantidade de variação que está associada aos dois níveis da hierarquia foi construído um modelo baseline (M1) a partir da ANOVA de efeitos aleatórios (designado por modelo nulo). O M1 não contém qualquer preditor ou covariável que explique o comportamento da variância dos dois níveis, tão pouco modela o efeito temporal no desenvolvimento coordenativo refletindo em cada um os quatro testes.

2) De seguida passamos a testar, sequencialmente, num conjunto de modelos que pretendem descrever as trajetórias temporais dos diferentes testes 
do KTK - desde modelos lineares a não lineares (a FIGURA 1 representa sequencialmente, módulos de trajetórias individuais e da diferenças entre crianças de natureza linear e não linear).

Só depois de ser identificado o modelo mais adequado para descrever a mudança coordenativa é que se consideraram efeitos aleatórios.

1 - Linear, trajetórias paralelas.

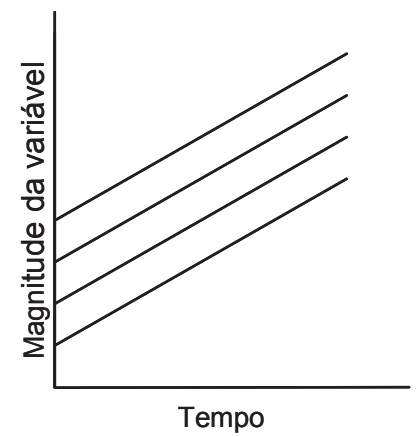

3 - Não linear, trajetórias paralelas.

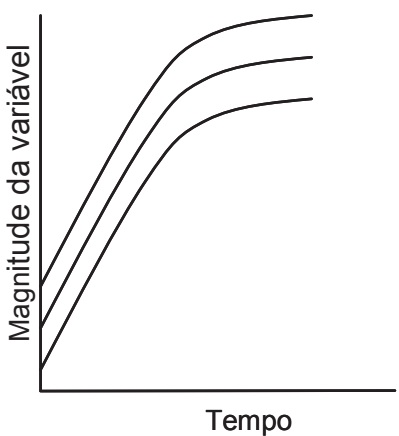

3) Foram testadas as covariáveis que se pensa estarem associadas às diferenças inter-individuais nas mudanças intra-individuais (IMC e AtFís).

4) Finalmente, foi analisado a diferença de trajetórias modais entre meninos e meninas. É esta estrutura sequencial de análise que se encontra sumariamente descrita na TABELA 3.

2 - Linear, trajetórias não paralelas.

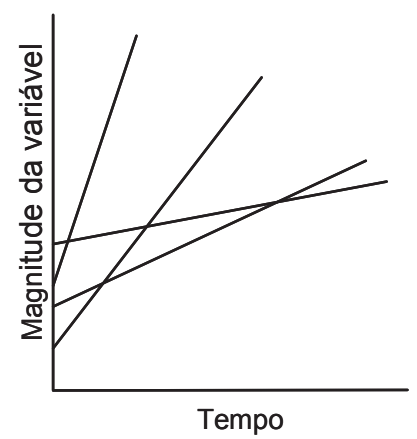

4 - Não linear, trajetórias não paralelas.

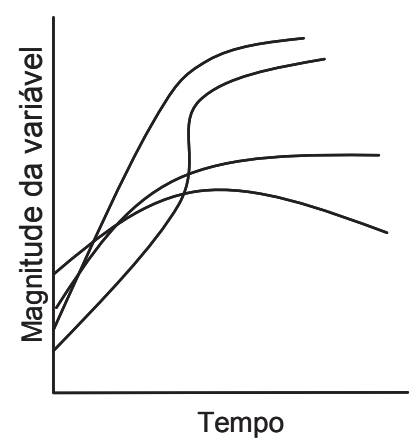

FIGURA 1 - Exemplos de quatro tipos de representação gráfica de trajetórias intra-individuais e diferenças inter-individuais.

\section{Resultados}

Os aspectos descritivos fundamentais das quatro provas (médias e desvios- padrão) nos quatro pontos no tempo estão naTABELA 1.

Nos quatro testes da bateria KTK (TABELA 1), em ambos os sexos e tal como esperado, percebese um incremento dos valores médios ao longo da idade $(\mathrm{p}<0,001)$. A magnitude do desvio-padrão, que indica a variação dos valores individuais $\mathrm{em}$ torno da média, revela diferenças inter-individuais marcantes em cada teste, sobretudo nos testes EDT,
SM e SL. O teste TL é o que apresenta menor variação nos valores em ambos os sexos.

Nos quatro testes da bateria KTK (TABELA 1), em ambos os sexos e tal como esperado, percebe-se um incremento dos valores médios ao longo da idade $(\mathrm{p}<$ 0,001). A magnitude do desvio-padrão, que indica a variaçâo dos valores individuais em torno da média, revela diferenças inter-individuais marcantes em cada teste, sobretudo nos testes EDT, SM e SL. O teste TL é o que apresenta menor variação nos valores em ambos os sexos. 
Os valores médios do IMC (TABELA 2) aumentaram com o passar dos anos em ambos os sexos, resultado mais do que esperado $(\mathrm{p}<0,01)$. Os valores médios dos níveis de AtFís, nos meninos, diminuíram da primeira para segunda avaliação e tornou a aumentar nos anos seguintes. É importante salientar que os valores dos desvios-padrão em torno da média indicam homogeneidade dos resultados da AtFís e do IMC em ambos os sexos (TABELA 2).

TABELA 1 - Medidas descritivas dos testes KTK, de meninos e meninas, nos quatro anos do estudo.

\begin{tabular}{|c|c|c|c|c|c|}
\hline \multicolumn{2}{|c|}{ Testes } & $\begin{array}{c}\text { EDT } \\
\mathrm{M} \pm \mathrm{dp}\end{array}$ & $\begin{array}{c}S L \\
M \pm d p\end{array}$ & $\begin{array}{c}S M \\
M \pm d p\end{array}$ & $\begin{array}{c}\text { TL } \\
M \pm d p\end{array}$ \\
\hline \multirow[t]{2}{*}{$1^{\text {o. }}$ ano } & $\sigma^{\pi}$ & $29,20 \pm 14,20$ & $31,34 \pm 9,72$ & $18,50 \pm 11,48$ & $15,07 \pm 2,68$ \\
\hline & q & $28,35 \pm 14,82$ & $28,30 \pm 8,74$ & $14,58 \pm 9,90$ & $13,87 \pm 2,54$ \\
\hline \multirow[t]{2}{*}{$2^{\text {o. }}$ ano } & $0^{\pi}$ & $37,06 \pm 14,08$ & $39,60 \pm 10,84$ & $27,18 \pm 15,71$ & $18,38 \pm 3,26$ \\
\hline & q & $36,07 \pm 14,19$ & $35,67 \pm 10,04$ & $22,99 \pm 13,41$ & $17,89 \pm 3,20$ \\
\hline \multirow[t]{2}{*}{$3^{\circ}$ ano } & $\sigma^{\pi}$ & $42,43 \pm 13,91$ & $43,94 \pm 14,07$ & $37,22 \pm 17,99$ & $20,68 \pm 3,39$ \\
\hline & $q$ & $40,18 \pm 11,78$ & $42,99 \pm 13,77$ & $34,67 \pm 17,13$ & $19,74 \pm 3,81$ \\
\hline \multirow[t]{2}{*}{$4^{\circ}$ ano } & $\sigma^{\pi}$ & $48,22 \pm 13,09$ & $53,63 \pm 13,89$ & $41,11 \pm 19,18$ & $20,92 \pm 3,75$ \\
\hline & q & $46,15 \pm 13,78$ & $53,44 \pm 12,12$ & $39,43 \pm 18,63$ & $20,16 \pm 3,44$ \\
\hline
\end{tabular}

EDT = Equilíbrio em deslocamento para trás; $\mathrm{SL}=$ Salto Lateral; $\mathrm{SM}=$ Salto Monopedal;

TL = Transposição Lateral.

TABELA 2 - Medidas descritivas do IMC e AtFís de meninos e meninas nos quatro anos do estudo.

\begin{tabular}{|c|c|c|c|c|c|}
\hline & & $1^{\circ}$ ano & $2^{\circ}$ ano & $3^{\circ}$ ano & $4^{\circ}$ ano \\
\hline & & $M \pm d p$ & $M \pm d p$ & $M \pm d p$ & $M \pm d p$ \\
\hline \multirow[t]{2}{*}{ IMC } & $\hat{\sigma}$ & $17,40 \pm 2,48$ & $17,41 \pm 2,86$ & $18,03 \pm 3,21$ & $18,71 \pm 3,61$ \\
\hline & q & $17,18 \pm 2,38$ & $17,18 \pm 2,72$ & $17,86 \pm 2,88$ & $18,62 \pm 3,30$ \\
\hline \multirow[t]{2}{*}{ AtFis } & $\widehat{0}$ & $52,19 \pm 31,68$ & $43,35 \pm 25,30$ & $47,95 \pm 22,93$ & $49,65 \pm 23,59$ \\
\hline & o & $41,81 \pm 31,94$ & $35,65 \pm 22,00$ & $34,90 \pm 30,13$ & $33,36 \pm 20,44$ \\
\hline
\end{tabular}

No TABELA 3 e na FIGURA 2 (modelação das figuras intra-individuais e diferenças inter-individuais) estão os resultados genéricos dos modelos para cada um dos testes da bateria KTK.

A opção pelo melhor modelo baseou-se nas reduçôes das Deviances sempre estatisticamente significativas $(\mathrm{p}<0,05)$.

Para todos os testes do KTK é saliente que o efeito do tempo se associa a prática de AtFís e ao IMC. No teste EDT, o tempo ${ }^{2}$ não foi significativo.

Em nenhum dos testes se verificou uma presença significativa do preditor do $2^{\circ}$ nível (gênero) i.e., meninos e meninas têm desempenhos paralelos e praticamente sobreponíveis em todos os testes. Não se confirma a presença de trajetórias coordenativas distintas nos dois sexos.

As representações gráficas da FIGURA 3 correspondem às trajetórias modais de cada prova, onde está salientada a influência da AtFís e do IMC em termos combinatórios do $1^{\circ}$ e $3^{\circ}$ quartis. Temos pois quatro trajetórias modais algo distintas, salientando a diversidade de mudança em cada uma das quatro provas, cujas estatísticas principais, e parâmetros mais importantes estão descritos na TABELA 4. 
DEUS, R.K.B.C. et al.

TABELA 3 - Resultados da análise sequencial dos modelos que melhor se ajustam aos dados.

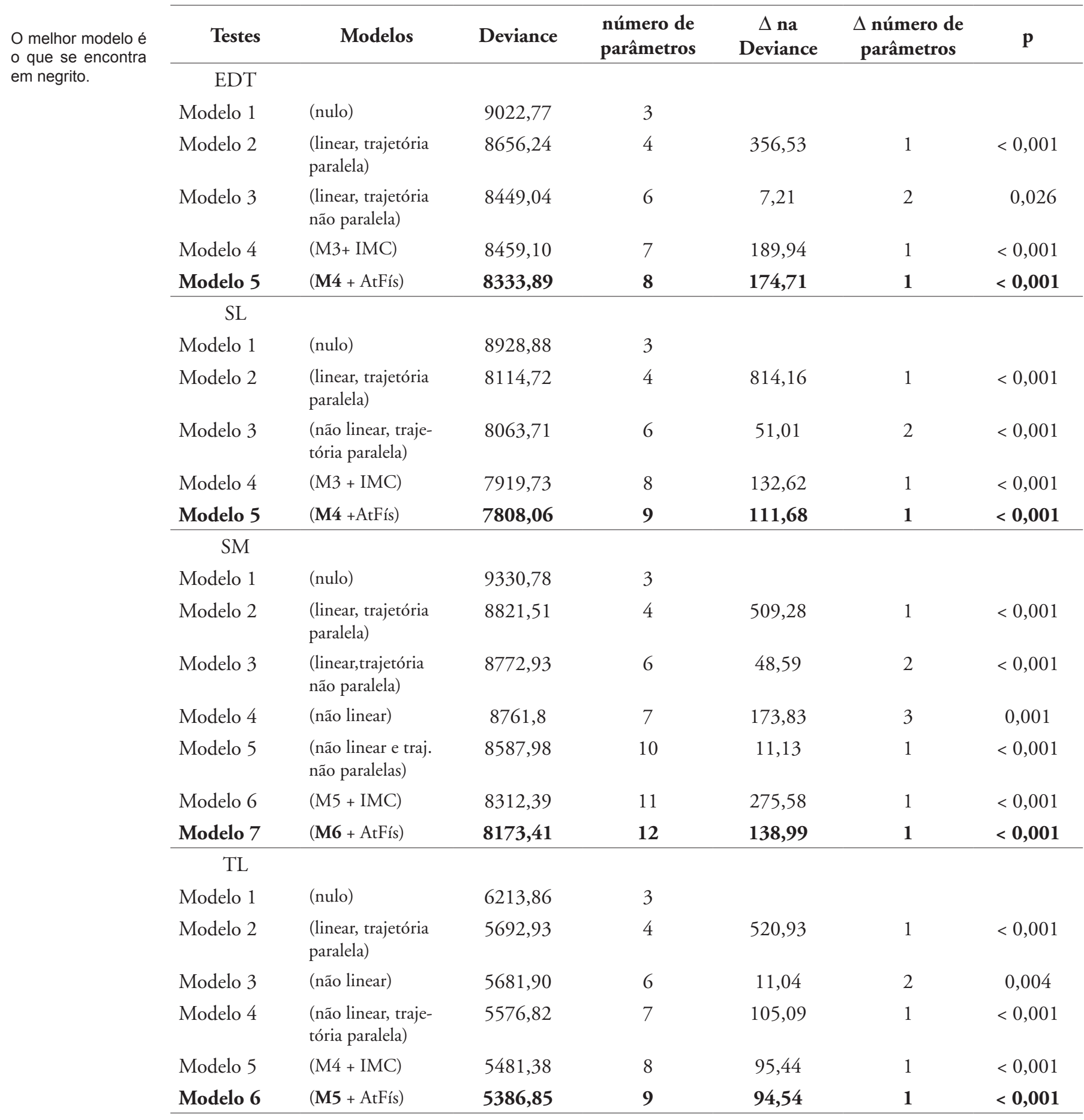

264 • Rev. bras. Educ. Fís. Esporte, São Paulo, v.24, n.2, p.259-73, abr./jun. 2010 
EDT

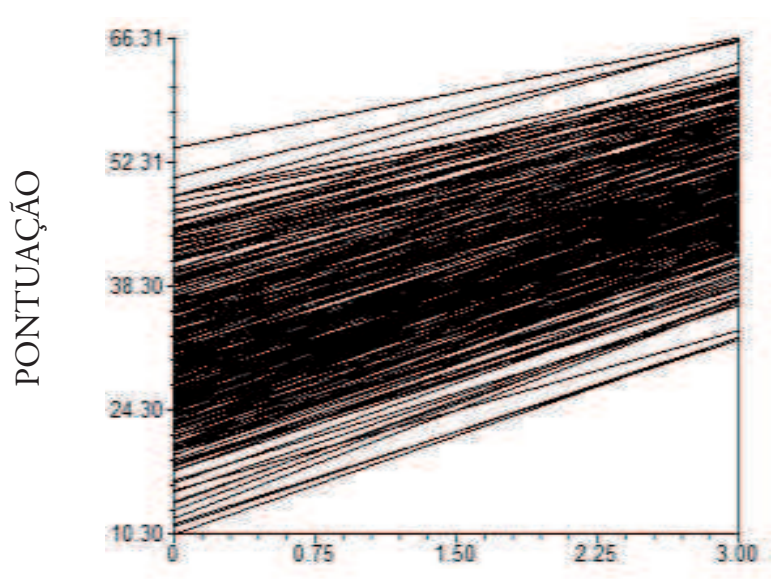

TEMPO

SM

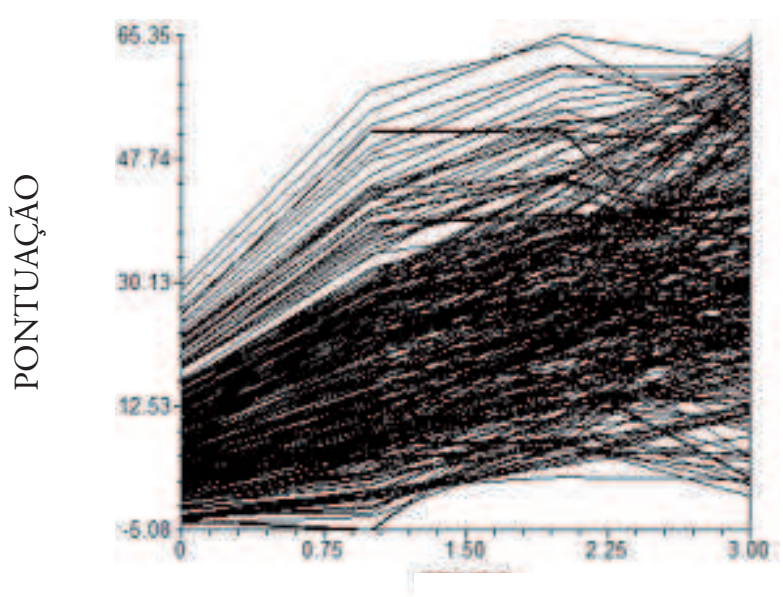

TEMPO
SL

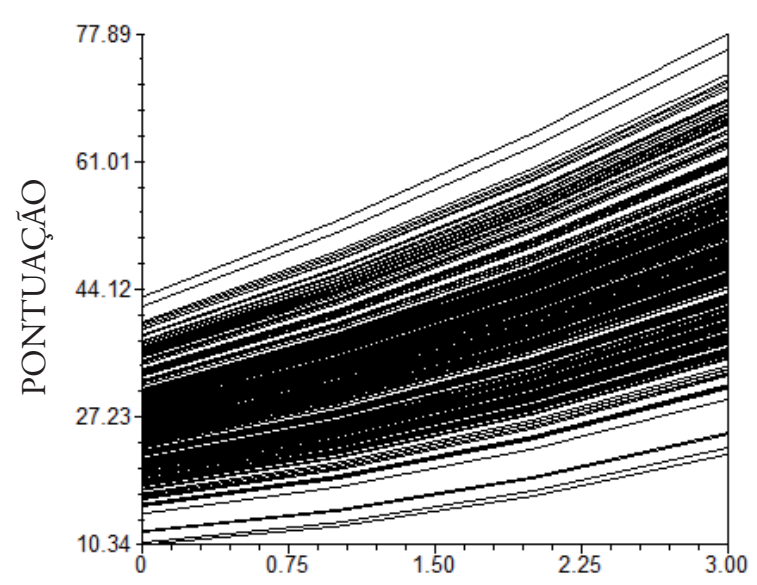

TEMPO

TL

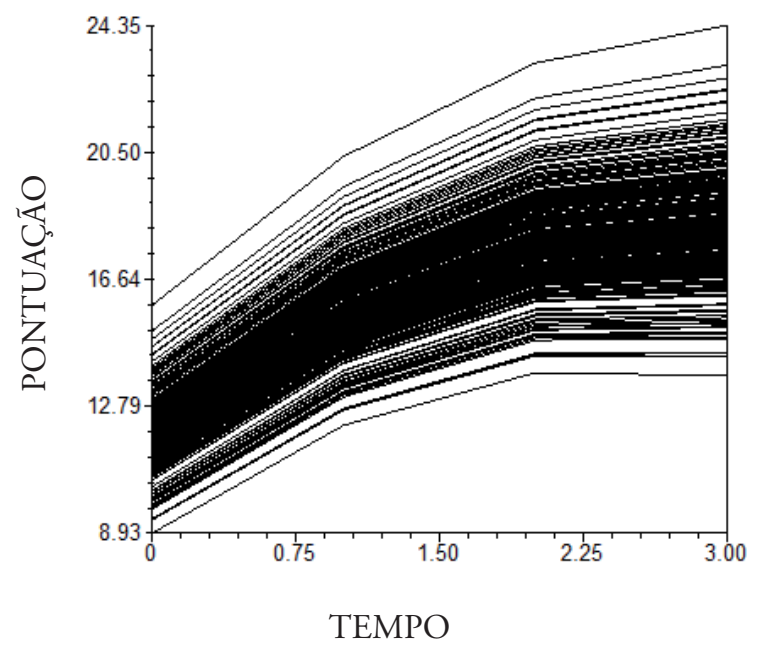

EDT = Equilíbrio em deslocamento para trás;

$\mathrm{SL}=$ Salto Lateral; $\mathrm{SM}=$ Salto Monopedal;

TL = Transposição Lateral.

FIGURA 2 -Trajetórias intra-individuais dos dados modelados em cada um dos testes da bateria KTK. 


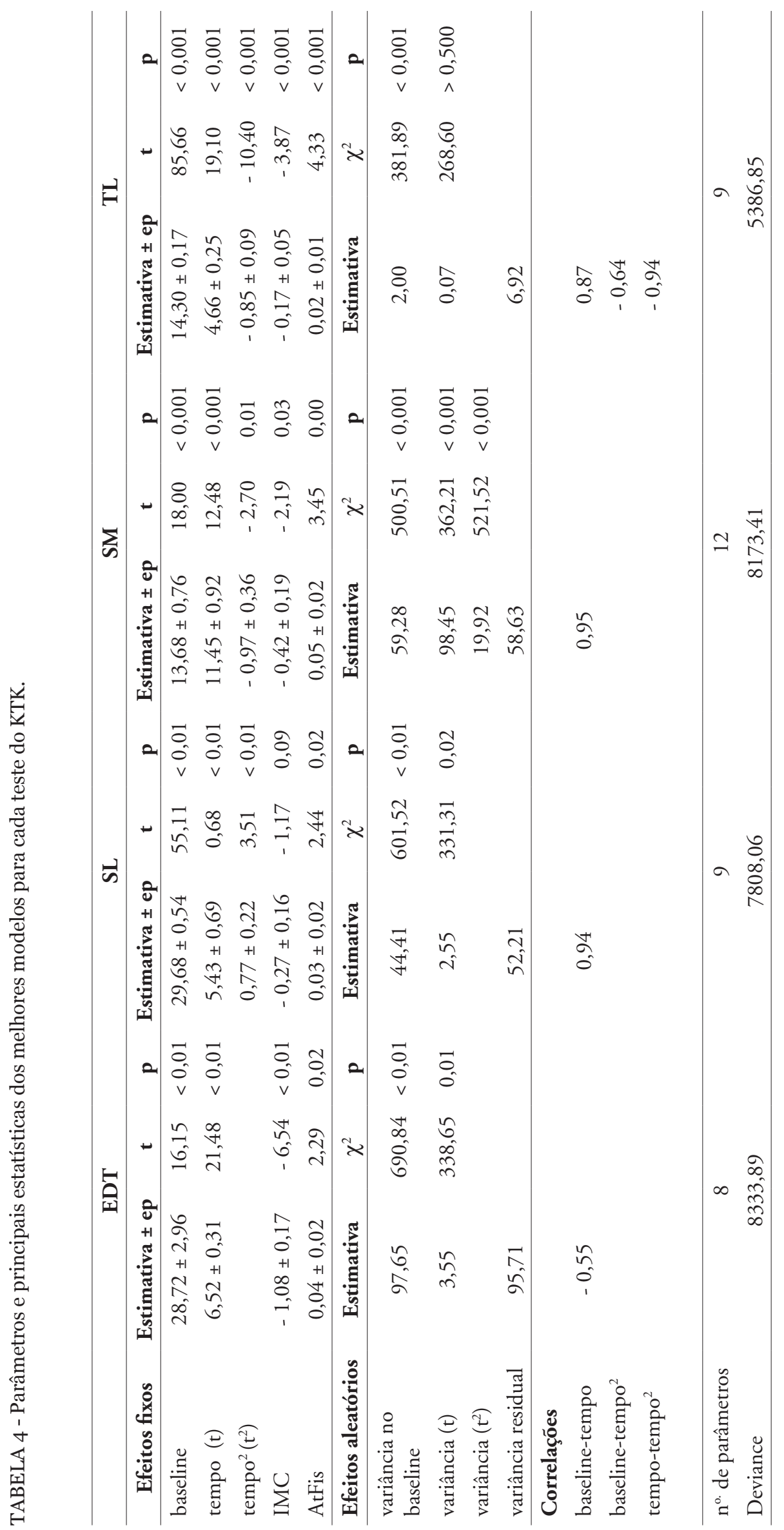

266 • Rev. bras. Educ. Fís. Esporte, São Paulo, v.24, n.2, p.259-73, abr./jun. 2010 
EDT

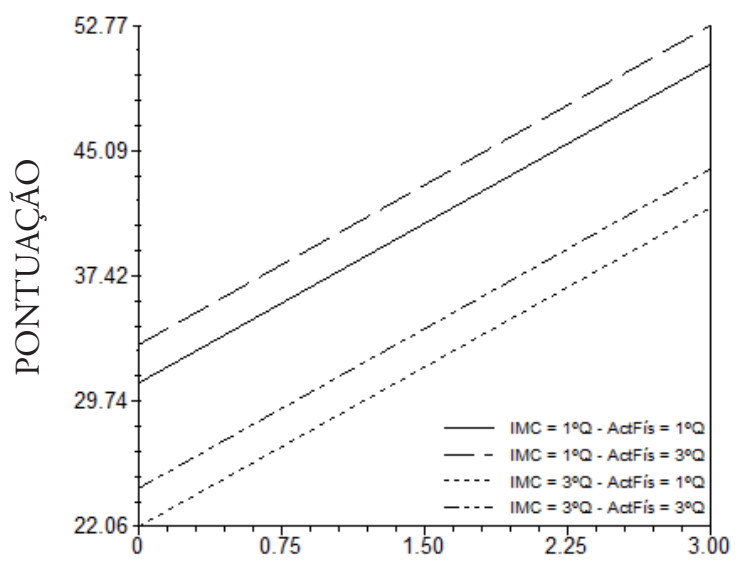

TEMPO

\section{SM}

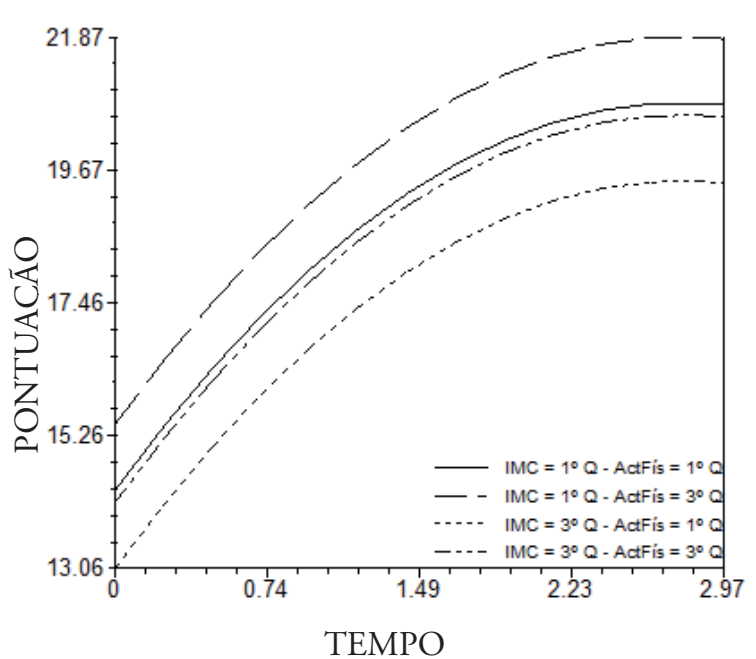

SL

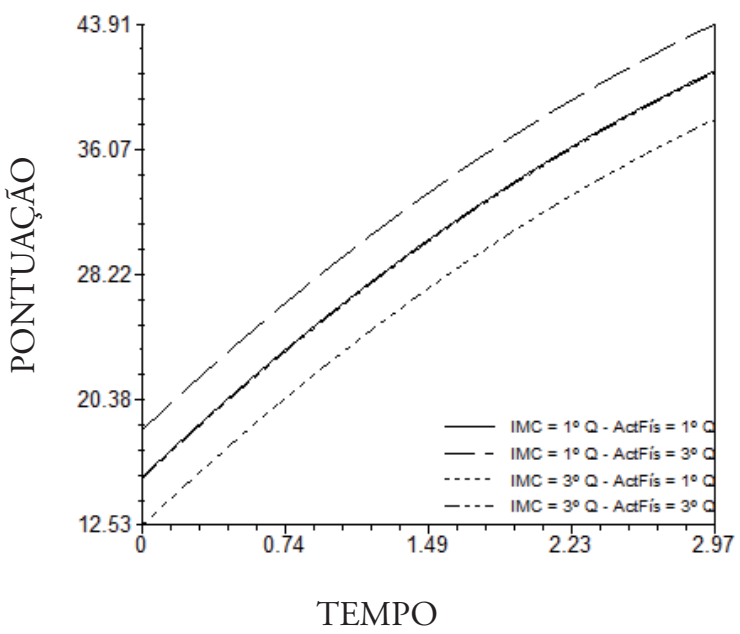

TL

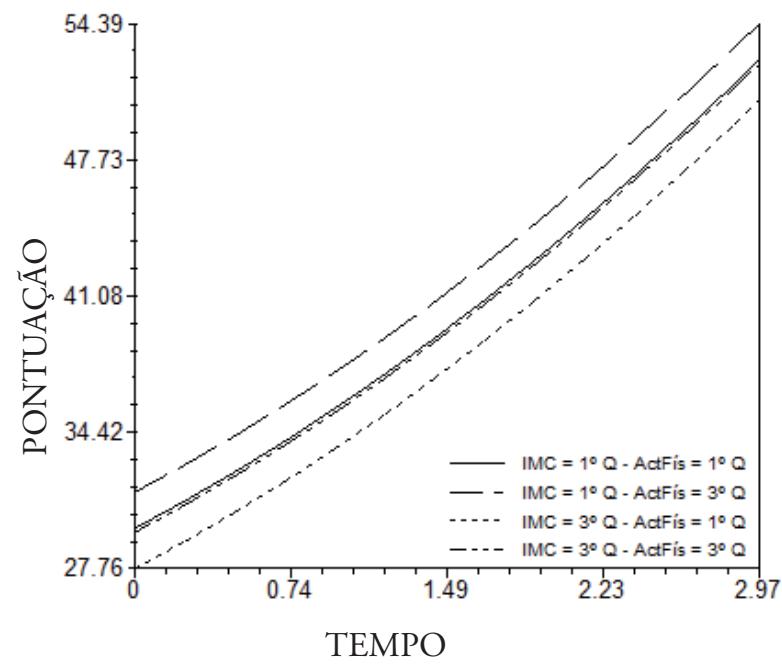

EDT = Equilíbrio em deslocamento para trás; $\mathrm{SL}=$ Salto Lateral; $\mathrm{SM}=$ Salto Monopedal; $\mathrm{TL}=$ Transposição Lateral.

FIGURA 3 - Trajetórias modais de cada teste do KTK em função do $1^{\circ}$ e $3^{\circ}$ quartil do IMC e AtFís.

$\mathrm{O}$ valor no baseline corresponde à média do desempenho no início do estudo $(47,80 \pm 2,86$ no EDT; $29,98 \pm 0,54$ no SL; $13,68 \pm 0,76$ no SM e $14,30 \pm 0,17$ no TL). É importante salientar a presença de variância inter-individual significativa $(\mathrm{p}<0,001)$ no início do estudo que expressa as diferenças entre sujeitos, i.e., a heterogeneidade de desempenho coordenativo no início da pesquisa (97,65 no ER; 44,41 no SL; 59,28 no SM e 2,00 no TL). A variável tempo (do inglês "time as a predictor of change") foi significativa em todos os testes, i. e., o aumento da idade implicou incrementos do desempenho anual em cada prova do KTK (EDT: 6,52 por ano; SL: 5,43 por ano; SM: 11,45 por ano e TL: 4,66 por ano). Foi necessário a presença de um polinômio do $2^{\circ}$ grau $\left(\right.$ tempo $\left.^{2}\right)$ para descrever as trajetórias curvilíneas do desempenho individual que mostrou ser estatisticamente significativo ( $\mathrm{p}<$ 0,05) nos testes SL, SM e TL.

Níveis mais elevados de AtFís implicaram aumentos significativos no desempenho da prova, sendo que o contrário aconteceu para o IMC - quanto maior o seu valor tanto menor o desempenho.

Finalmente importa realçar a magnitude e significado estatístico das correlaçôes entre os parâmetros descritores da mudança e as diferenças inter-individuais. Assim, por exemplo, na prova EDT quanto maior for $\mathrm{o}$ valor no baseline menor serão os ganhos anuais ( $\mathrm{r}$ $=-0,55)$. O contrário acontece na prova TL, em que um maior valor de partida implica maior desempenho anual na fase linear $(r=0,87)$ e menor declínio de prestação coordenativa na fase não-linear $(r=-0,64)$. 


\section{Discussão}

Os grandes propósitos deste estudo foram a descrição e interpretação de diferentes aspectos da mudança do desenvolvimento da CoM de crianças do $1^{\circ} \mathrm{CEB}$ a partir de um delineamento longitudinal puro na primeira coorte do estudo da RAA (MAIA et al., 2003). Tanto quanto julgamos saber, é a primeira vez que dados longitudinais do KTK são explorados de uma forma bem distinta da apresentada por WillimCZiK (1980), bem como fora do quadro habitual da interpretação clínico-pedagógica que se faz do quociente motor $(\mathrm{QM})$. Face à complexidade da análise e dos problemas em estudo, parece-nos importante situar a discussão dos resultados em dois planos: 1) o metodológico e o 2) substantivo.

Vejamos em primeiro lugar as questóes metodológicas. Os dados disponíveis da pesquisa com a bateria de testes KTK são de natureza experimental ou quasiexperimental (LOPES, 1997; MOTA, 1991; ZAICHKOWSKY, ZAICHKOWSKY \& MARTINEK, 1978), transversal (Andrade, 1996; Bustamante, 2007; Gomes, 1996) e muito raramente longitudinal (KIPHARD \& SCHILLING, 1974; WillimcZiK, 1980). A grande preocupação deste tipo de investigação centrada nas variáveis do KTK é, essencialmente, descrever diferenças de médias entre sexos em diferentes idades, e/ou a eficácia de programas de intervenção. Em nosso entender trata-se de uma visão importante, mas parcial, porque não considera outras questôes relevantes do ponto de vista desenvolvimentista (Foster \& KaliL, 2008; RAUDENBush et. al., 2004), tão pouco explora todas as potencialidades dos dados disponíveis, não obstante a versatilidade do modelo linear plasmado na ANOVA. $\mathrm{O}$ recurso à modelação hierárquica viabilizada pelo "software" estatístico HLM 6.0 permite, pela sua versatilidade e extensão informativa baseada no teste sequencial de modelos de complexidade crescente, uma interpretação de dados longitudinais bem mais atraente e esclarecedora (MAIA et al., 2007).

A bateria de testes KTK foi construída com propósitos descritivos e clínicos, desaguando no cálculo do QM e a sua classificação. Não obstante o seu valor para a época, contexto sócio-cultural e educativo alemão, o fato incontroverso é que não se dispõe de informação sólida acerca da validade dos valores de corte do QM tão pouco da sua validade transcultural para a RAA. Acrescenta-se o fato de não se conhecer o significado clínico e pedagógico da classificação proposta pelos autores alemães. Decorre daqui a opção pelo uso individual dos valores de cada prova do KTK.
As questões associadas à dimensão amostral, sua representatividade e capacidade em gerar parâmetros que descrevam aspectos fundamentais dos problemas em apreço são comuns na literatura desenvolvimentista ou epidemiológica (ver a este respeito o número especial da International Journal of Sports Medicine dedicada à temática "Problems and Solutions in Longitudinal Research", v.18, 1997. Supl.3). Por exemplo, o estudo de WilLIMCZIK (1980) amostrou 705 crianças no início do estudo e finalizou com 399 crianças. A pesquisa presente recorreu a um processo de amostragem aleatória e proporcional de quatro ilhas que têm aproximadamente $99 \%$ do universo escolar. Não obstante a dimensão do presente estudo ser inferior à do autor alemão, tem poder suficiente para testar o significado substantivo dos problemas em apreço (Spybrook, Raudenbush, Liu \& Congdon, 2006).

$\mathrm{O}$ último aspecto metodológico refere-se ao uso do questionário de Godin e SHEPHARD (1985) para marcar níveis de AtFís das crianças. Apesar deste e qualquer outro questionário apresentar dificuldades inerentes à natureza subjetiva das respostas, requer um tratamento cuidadoso sobretudo em relação à qualidade da informação obtida. Este inventário é simples, válido e fiável em crianças (SAllis, Buono, Roby, Micale \& Nelson, 1993). Por exemplo, Scerpella, Tuladhar e Kamaley (2002) realizaram um estudo para validar o questionário Godin e SHEPHARD em ginastas do sexo feminino (sete a 11 anos) e encontrou correlações moderadas $($ rho $=0,38$ a 0,42$)$ entre este questionário e outros instrumentos utilizados para avaliar a AtFís (PACI, Caltrac e Self-reported Activity hr/semana) apresentaram uma correlação moderada. FALK, Portal, Tiktinsky, Weinstein, Constantini e MARTINOWITZ (2000) avaliou aspectos da potência aeróbia e muscular de meninos hemofílicos (12,0 $\pm 3,17$ anos) e comparou com meninos saudáveis $(11,9 \pm 2,8$ anos). Serviu-se do questionário GS para diferenciar os seus níveis de AtFís. Numa pesquisa sobre a consistência do questionário de GS, Koo e ROHAN (1999) comparou a fiabilidade de quatro questionários da AtFís habitual. No caso vertente do GS, o valor do teste-reteste foi de $r=0,53$. Este resultado sugere que o questionário providencia medidas simples e práticas de AtFís habitual de crianças e adolescentes. Em Portugal, SousA (2005) encontrou valores do coeficiente de correlação intraclasse para o questionário GS entre 0,88 e 0,90 o 
que certifica um valor baixíssimo de variância-erro. No mesmo sentido são os resultados providenciados por MAIA e Lopes (2002) num trabalho realizado na RAA com crianças dos seis aos 10 anos, em que a estimativa de fiabilidade do questionário de GS se situa entre 0,75 e 0,88 .

Vejamos agora aspectos substantivos dos resultados. Foi verificado um incremento nas médias dos quatro testes, tal como seria de esperar face ao próprio processo de crescimento e desenvolvimento das crianças. Comportamento semelhante ocorre nas mais diversas aptidões face ao desenvolvimento motor generalizado que ocorre ao longo da idade (HAYWOOD \& GETCHELL, 2005; Lopes et al., 2003; MALINA, 2005). Resultados equivalentes aos do presente estudo foram encontrados por KipHard e SChilling (1976), WillimcziK (1980) e LOPES (1997). Em todos os testes as meninas apresentaram valores inferiores aos meninos. Também WiLLIMCZIK (1980) verificou este dimorfismo sexual, embora nas meninas, e apenas aos seis anos, houvesse uma vantagem no desempenho coordenativo. Devemos ressaltar que o estudo de WiLLIMCZIK (1980), apesar de ser também de natureza longitudinal centra sua analise somente no QM.

Os valores dos desvios-padrão são de magnitude elevada no teste SM em ambos os sexos, sugerindo uma tendência de incremento progressivo de diferenças do desempenho entre crianças nos quatro momentos de avaliação $(9,90$ a 18,63 e 11,48 a 19,18 nas meninas e meninos, respectivamente). Este efeito é conhecido por "fan out", dado sugerir a abertura de um leque reproduzido pelos aumentos de variância ao longo de quatro anos de estudo. Este incremento na variabilidade inter-individual, reflete trajetórias distintas de desenvolvimento da CoM entre as crianças ao longo dos quatro anos. No teste TL, a magnitude dos valores é mais reduzida $(2,54$ a 3,44 e 2,68 a 3,75 nas meninas e meninos, respectivamente), mas sugere um comportamento semelhante. É evidente que existem crianças que na sua idade expressam características no seu desenvolvimento coordenativo que são de crianças em idade mas avançadas e viceversa. Segundo Bruner (1965) citado por Lopes (1997) a criança está sempre pronta para algum tipo de experiências, mas a seleção e provisão de estímulos que desencadeiem a resposta desejada são da responsabilidade do professor. A heterogeneidade encontrada nos valores dos desvios-padrão dos testes, em função da idade, reclama uma atenção elevada no planejamento das aulas de Educação Física, especialmente na sua estrutura didática-metodológica em cada ano de escolaridade (LOPES, 1997).
Os resultados mostrados na FIGURA 3 são de grande relevância, e não conhecemos qualquer pesquisa neste sentido, ressaltamos aqui a trajetórias modal para cada prova do KTK em termos combinatórios de quartis. Temos para cada teste quatro trajectórias distintas que salientam a diversidade de mudança. $\mathrm{O}$ estudo da mudança requer que se olhe com atenção para o desenvolvimento como um fenômeno dinâmico e não como um fenômeno estático (LOPES, 1997). Estamos a considerar uma mesma distribuição de crianças que apresenta trajetórias distintas para cada teste. De acordo com as representações da FIGURA 3 é evidente o incremento progressivo das trajetórias nos testes durante os quatro anos. $\mathrm{O}$ teste EDT foi o único que apresentou uma trajetória linear, que não ocorreu nos outros testes e, demonstrou em seus resultados que quanto maior for o valor de partida menor são os ganhos anuais $(\mathrm{r}=$ $-0,55)$. Por outro lado, os testes SL e SM mostraram que quanto maior for o valor na baseline maiores são os ganhos anuais $(\mathrm{r}=0,94$ e 0,95 , respectivamente), considerando que, no SL é notório um efeito de incremento positivo distinto dos outros testes. No teste TL parece existir um efeito de travagem a partir da terceira avaliação. De acordo com os resultados da TABELA 4, quanto maior for o seu valor de partida maior será o desempenho na fase linear $(r=0,87)$ e menor declínio na fase não-linear $(r=-0,64)$; as crianças que têm maiores performances no baseline são os que têm menor efeito de travagem desenvolvimentista aos 10 anos de idade ( $\mathrm{r}$ $=-0,94)$. Na generalidade, os resultados evidenciam que as competências coordenativas dos testes apresentam trajetórias distintas.

Neste sentido, quando foram modelados os resultados do KTK com o IMC e o nível de AtFís das crianças os melhores valores foram observados para as crianças que estavam no $1^{\circ}$ quartil do IMC e no $3^{\circ}$ quartil da AtFís; em segundo lugar aparecem as crianças que estão no $1^{\circ}$ quartil do IMC e no $1^{\circ} \mathrm{da}$ AtFís, de seguida estão as do $3^{\circ}$ quartil de ambos indicadores e por último as que estão $3^{\circ}$ quartil no IMC e no $1^{\circ}$ da AtFís (FIGURA 3).

$\mathrm{O}$ teste EDT apresentou um crescimento linear durante os quatro anos, com um ganho anual de 6,52 pontos. Há uma grande proximidade das trajetórias das crianças com os melhores resultados $\left(1^{\circ} \mathrm{Q}\right.$ $\mathrm{IMC} / 3^{\circ} \mathrm{Q}$ AtFís) e as que estão no $1^{\circ} \mathrm{Q}$ IMC e $1^{\circ} \mathrm{Q}$ AtFís (FIGURA 3). Por outro lado, as crianças que estão no $3^{\circ}$ quartil de ambos estão próximas das que estão no $3^{\circ} \mathrm{Q} \mathrm{IMC} / 1^{\circ}$ Q AtFís, porém, estão distantes das que têm os melhores resultados. Não podemos deixar de evidenciar que, quanto maior o valor na baseline maior é o seu desempenho anual. Esses 
resultados revelam que o IMC adequado é um fator essencial para o bom desempenho desta prova. De fato, a realização deste teste exige o deslocamento do centro de gravidade de uma forma equilibrada o que pode estar a penalizar as crianças mais pesadas. GRAF et al. (2004) constatou uma correlação inversa entre o IMC e o QM das crianças, ou seja, as crianças com sobrepeso e obesidade tiveram desempenho inferior quando comparadas com as crianças normoponderais e com baixo peso. O alto teor de gordura corporal possivelmente traz consequências negativas no desempenho dos testes coordenativos (GRAF et al., 2005).

Nos testes SM, TL e SL as trajetórias de mudança de natureza não-linear na sua forma. As crianças que se encontram no $3^{\circ} \mathrm{Q} I M C / 1^{\circ} \mathrm{Q}$ AtFís têm trajetórias praticamente sobrepostas as que estão no $3^{\circ} \mathrm{Q} I M C / 3^{\circ} \mathrm{Q}$ AtFís. Estes resultados sugerem alguma dependência coordenativa os níveis de AtFís para que ocorra um bom desempenho nestas provas. Como o desenvolvimento das capacidades coordenativas depende da quantidade e da qualidade de experiências motoras da criança (HIRTZ \& SCHIELK, 1986), uma das grandes preocupações educativas deveria situar ao nível das aulas de Educação Física.

O $1^{\circ}$ CEB é um período relevante para o desenvolvimento coordenativo. Os resultados disponíveis mostram a heterogeneidade da capacidade de desenvolvimento da CoM que reclama uma atenção

\section{Conclusão}

De acordo com os propósitos delineados para este estudo, podemos concluir que: 1) em ambos os sexos e ao longo dos quatro anos foi observado um incremento significativo dos valores médios de todas as provas constituintes da bateria KTK, sendo evidente um aumento da variabilidade interindividual em termos coordenativos. Os resultados vêm reafirmar o direito à diferença e reclamam o entendimento do seu significado em termos pedagógicos por todos os professores de Educação redobrada por parte dos professores de EF, pois duas crianças com a mesma idade cronológica podem apresentar níveis de desenvolvimento coordenativo distintos, ou seja, enquanto uma das crianças consegue realizar tarefas consideradas avançadas para a sua idade, a outra pode apenas conseguir tal desempenho com ajuda adicional externa.

A escola primária é essencial no processo de desenvolvimento e refinamento das capacidades coordenativas que serão fundamentais nos anos seguintes de escolaridade. Este estudo vem salientar que a escola é o local com a maior responsabilidade educativa. Nela se implementam hábitos de vida ativa e esportiva das crianças e, as aulas de EF tê, entre outras, a missão de conseguir que os alunos possuam uma elevada capacidade funcional, apresenta para cada idade, por forma a procurar adaptações rápidas e proveitosas nas mais diversas situaçôes motoras, a que se associam aquisições de novas habilidades (Hirtz \& Holtz, 1987). Contudo, uma atenção especial deve ser dada ao planejamento das aulas, especialmente à sua estrutura didática-metodológica em cada ano de escolaridade (LOPES et al., 2003), de modo que as crianças possam alcançar níveis coordenativos adequados para a sua idade. Neste sentido, avaliações periódicas dos níveis de CoM são essenciais para um planejamento mais consistente das aulas de acordo com as necessidades das crianças.

Física; 2) não houve diferenças significativas entre os meninos e as meninas nas suas trajetórias modais; 3 ) níveis mais elevados de AtFís implicaram aumentos significativos no desempenho de prova, sendo que o contrário aconteceu para o IMC, quanto maior o seu valor tanto menor o desempenho.

Os resultados disponíveis reafirmam a necessidade de estruturas didático-metodológicas adaptadas em cada ano de escolaridade para que as crianças alcancem níveis adequados de CoM para a sua idade. 


\begin{abstract}
Longetudinal modeling of motor coordination levels of children aged six to 10 years of age from the Autonomous Region of Azores, Portugal

This study aims at: 1) describing models for developmental changes and interindividual differences in gross motor coordination (CoM); 2) verify the presence of gender effects; 3 ) and test the importance of BMI and physical activity level in motor coordination (CoM) development. Sample size comprises 285 children ( 143 boys and 142 girls). Motor coordination was evaluated with the test battery "Körperkoordinationstest für Kinder" (KTK) consisting of four tests: backward balance (ER), jumping sideways (SL), hopping on one leg (SM), and shifting platforms (TL). Godin and Shephard's questionnaire was used to evaluate children's physical activity level. Basic statistics were computed in SPSS. Intraindividual changes and interindividual differences were estimated within the framework of multilevel modeling using the HLM software, version 6. Mean significant changes were noticed on both genders in $\mathrm{CoM}, \mathrm{BMI}$ and physical activity. Best CoM values were found for children in the 1st quartile of BMI and 3rd quartile of physical activity. These results call for a closer attention to novel approaches of didactical issues in the preparation of physical education classes, so that all children may achieve adequate levels of success according to their age.
\end{abstract}

UNITERMS: Motor coordination; Children; Longitudinal; Hierarchical modeling.

\title{
Nota
}

BRUNER, J. The process of education. Cambridge: Havard University, 1965.

\section{Referências}

ANDRADE, M.J.L.A. Coordenação motora: estudo em crianças do $1^{\circ}$ Ciclo Básico na Região Autónoma da Madeira. 1996. Tese (Mestrado) - Faculdade de Desporto, Universidade do Porto, Porto, 1996.

BUSTAMANTE, A. Análisis interactivo de la coordinación motora, actividad física y del índice de masa corporal en escolares peruanos: estudio transversal en niños de ambos sexos de los 6 a los 11 años de edad. 2007. Tese (Mestrado) - Faculdade de Desporto, Universidade do Porto, Porto, 2007.

FALK, B.; PORTAL, S.; TIKTINSKY, R.; WEINSTEIN, Y.; CONSTANTINI, N.; MARTINOWITZ, Y. Anaerobic power and muscle strenth in young hemophilia patientes. Medicine and Science in Sports and Exercise, Madison, v.32, p.52-7, 2000. FOSTER, E.M.; KALIL, A. New methods for new questions: obstacles and opportunities. Desenvolopmental Psychology, Washington, v.44, n.2, p.301-4, 2008. Disponível em: <http://www.unc.edu/ -emfoster/papers/newmethods.pdf . Acesso em: 7 jun. 2008.

GODIN, G.; SHEPHARD, R.J. A simple method to assess exercise behavior in the community. Canadian Journal of Applied Sport Sciences, Champaign, v.10, p.141-6, 1985.

GOLDSTEIN, H. Multilevel models in educational and social research. London: Griffin, 1987.

Multilevel statistical models. 2nd. ed. London: Edward Arnold, 1995.

GOMES, M.P.B.B. Coordenaçáo, aptidão física e variáveis do envolvimento: estudo em crianças do $1^{\circ}$ ciclo de ensino de duas freguesias do concelho de Matosinhos. 1996. Tese (Doutorado) - Faculdade de Desporto, Universidade do Porto, Porto, 1996. GRAF, C.; KOCH, B.; FALKOWSKI, G.; JOUCK, S.; CHRIST, H.; STAUENMAIER, K.; BJARNASON-WEHRENS, B.; TOKARSKI, W.; DORDEL, S.; PREDEL, H.G. Effects of a school-based intervation on BMI and motor abilities in childhood. Journal of Sports Science and Medicine, Bursa, v.4, p.291-9, 2005.

GRAF, C.; KOCH, B.; KRETSCHMANN-KANDEL, E.; FALKOWSKI, G.; CHRIST, H.; COBURGER, S.; LEHMACHER, W.; BJARNASON-WEHRENS, R.; PLATEN, P.; TOKARSKI,W.; PREDEL, H.G.; DORDEL, S. Correlation between BMI, leisure habits and motor abilities in childhood (CHILT-Project). International Journal of Obesity, London, v.28, p.22-6, 2004. 
HAYWOOD, K.M.; GETCHELL, N. Life span motor development. 4th ed. Champaign: Human Kinetics, 2005. HIRTZ, P.; HOLTZ, D. Como aperfeiçoar as capacidades coordenativas: exemplos concretos. Horizonte, Lisboa, v. 17, p.166-71, 1987. HIRTZ, P.; SCHIELK, E. O desenvolvimento das capacidades coordinativas nas crianças, nos adolescentes e nos jovens adultos. Horizonte, Lisboa, v.15, p.83-8, 1986.

KIPHARD, B.J.; SCHILLING, F. Körperkoordinations Test für Kinder: Beltz Test GmbH. Weinheim: [s.ed.], 1974. KOO, M.M.; ROHAN, T.E. Comparison of four habitual physical activity questionaires in girls aged 7-15yr. Medicine and Science in Sports and Exercise, Madison, v.31, n.3, p.421-7, 1999.

LOPES, V.; MAIA, J.A.; SILVA, R.; SEABRA, A.; MORAIS, F. Estudo do nível de desenvolvimento da coordenação motora da população escolar (6 a 10 anos de idade) da Região Autónoma dos Açores. Revista Portuguesa de Ciências do Desporto, Porto, v.3, n.1, p.47-60, 2003.

LOPES, V.P. Análise dos efeitos de dois programas distintos de educação física na expressão da aptidão física, coordenação motora e habilidades motoras em crianças do ensino primário. 1997. Tese (Doutorado) - Faculdade de Desporto, Universidade do Porto, Porto, 1997.

LOPES, V.P.; MAIA, J.A.R. Efeitos do ensino no desenvolvimento da capacidade de coordenação corporal em crianças de 8 anos de idade. Revista Paulista de Educação Física, São Paulo, v.11, n.1, p.40-8, 1997.

MAIA, J.A.; GARGANTA, R.; SEABRA, A.; LOPES, V.P.; VINAGRE, J.; FREITAS, D.L.; PRISTA, A.; MEIRA, C. Dados longitudinais e modelação hierárquica: um tutorial de investigadores das ciências do desporto. Revista Brasileira de Cineantropometria e Desempenho Humano, Florianópolis, v.7, n.2, p.94-108, 2005.

MAIA, J.A.; SOUSA, M.A.; FERMINO, R.C.; SEABRA, A.; SILVA, S.; SILVA, R.G.; BUSTAMANTE, A.; LOPES, V.P.; PRISTA, A.; FREITAS, D. Análise e interpretação dos níveis de actividade física de crianças: um tutorial baseado na modelação hierárquica ou multinivel. Revista Brasileira de Cineantropometria e Desempenho Humano, Florianópolis, v.9, n.4, p.424-35, 2007. MAIA, J.A.R.; LOPES, V.P. Estudo do crescimento somático, aptidão física, actividade física e capacidade de coordenação corporal de crianças do $1^{\circ}$ ciclo do ensino básico da Região Autônoma dos Açores. Porto: DREFD/FADEUP, 2002.

Estudo do crescimento somático, aptidáo física, actividade física e capacidade de coordenação corporal de crianças do primeiro ciclo do ensino básico da Regiáo Autónoma dos Açores. Porto: DREFRAA /Faculdade de Desporto, Universidade do Porto, 2003.

MAIA, J.A.R.; LOPES, V.P.; SILVA, R.G.; SEABRA, A.; FERREIRA, J.V.; CARDOSO, M.V. Modelação hierárquica ou multinível. Uma metodologia estatística e um instrumento útil de pensamento na investigação em Ciências do Desporto.

Revista Portuguesa de Ciências do Desporto, Porto, v.3, n.1, p.92-107, 2003.

MALINA, R. Variation in body composition associated with sex and ethnicity. In: HEYMSFIELD, S.B. et al. Human body composition. Champaign: Human Kinetics, 2005. p.271-98.

MOTA, J.A.P.S. Contributo para o desenvolvimento de programas de aulas suplementares de educaçáo física: estudo experimental em crianças com insuficiências de rendimento motor. 1991. Tese (Doutorado) - Faculdade de Desporto, Universidade do Porto, Porto, 1991. RAUDENBUSH, S.; BRYK, A.; CHEONG, Y.F.; CONGDON, R. HLM 6: Hierarchical linear \& non-linear modeling. Chicago: Scientific Software International, 2004.

RAUDENBUSH, S.W.; BHUMIRAT, C. The distribution of resources for primary education and its consequences for education achievement in Thailand. International Journal of Educational Research, Oxford, v.17, n.2, p.143-64, 1992. SALLIS, J.F.; BUONO, M.J.; ROBY, J.J.; MICALE, F.G.; NELSON, J.A. Seven-day recall and other physical activity self-reports in children and adolescents. Medicine and Science in Sports and Exercise, Madison, v.25, p.99-108, 1993. SCERPELLA, J.A.; TULADHAR, P.; KAMALEY, A. Validation of the Godin-Shephard questionnaire in prepubertal girls. Medicine and Science in Sports and Exercise, Madison, v.5, p.845-50, 2002.

SILVA, G.A.S. Análise da coordenaçáo ampla (grossa) em crianças de 7 a 10 anos. 1989. Tese (Mestrado) - Universidade de São Paulo, São Paulo, 1989.

SOUSA, M.A.; MAIA, J.A.R. Crescimento somático, actividade física e aptidão física associada à saúde: um estudo populacional nas crianças do $1^{\circ}$ ciclo do ensino básico do concelho de Amarante. Amarante: Edições da Câmara Municipal de Amarante/FADEUP, 2005.

SPYBROOK, J.; RAUDENBUSH, S.W.; LIU, X.; CONGDON, R. Optimal design for longitudinal and multilevel research: documentation for the "Optimal Design" Software. 2006. Disponível em: <http://sitemaker.umich.edu/group based/optimal_design_software>. Acesso em: 23 jun. 2008.

WILLIMCZIK, K. Development of motor control capability (body coordination) of 6-to 10-year-old children: results of a longitudinal study. In: OSTYN, M.; BEUNEN, G.; SIMONS, J. (Eds.). Kinanthropometry II. Baltimore: University Park Press, 1980. p.328-46. 
ZAICHKOWSKY, L.D.; ZAICHKOWSKY, L.B.; MARTINEK, T,J. Physical activity, motor development age and sex differences. In: LANDRY, F.; ORBAN, W.D.R. (Eds.). Motor learning, sport psychology, pedagogy and didactics of physical activity. Miami: Symposia Specialists, 1978.

\section{Agradecimentos}

À Direção Regional de Desporto da Região Autônoma dos Açores, especialmente ao seu Diretor, Dr. Rui Santos, pelo seu comprometimento e financiamento do projeto Crescimento e Desenvolvimento de crianças e jovens açorianos.

Aos revisores anônimos cujas sugestóes permitiram melhorar o texto.

José António Ribeiro Maia

ENDEREÇO Laboratório de Cineantropometria

Faculdade de Desporto

Universidade do Porto

R. Dr. Plácido Costa, 91

Recebido para publicação: 07/07/2008

Revisado em: 19/03/2009

Aceito: 02/12/2009

e-mail: jmaia@fade.up.pt 